\title{
Reduced c-Fos expression in orexin neurons of the lateral hypothalamic area and the locus coeruleus following injection of spinosin into mice
}

\author{
J.-P. Zhang ${ }^{1}$, D.-Q. Liao², L. Li ${ }^{3}$ L. Chu \\ ${ }^{1}$ Department of Anatomy, Histology and Embryology, Basic Medical College, Zhejiang Chinese Medical University, \\ Hangzhou, China \\ ${ }^{2}$ Clinical Practice Teaching Centre, Zhejiang Chinese Medical University, Hangzhou, China \\ ${ }^{3}$ Department of Physiology, Basic Medical College, Zhejiang Chinese Medical University, Hangzhou, China
}

[Received: 1 August 2019; Accepted: 12 October 2019]

\begin{abstract}
Background: Spinosin, a major component of Samen Ziziphi spinosae, has been shown to modulate sedation and hypnosis; however, the underlying neuronal mechanisms of its stimulatory effects remain unclear.

Materials and methods: In the present study, we injected spinosin $(15 \mathrm{mg} / \mathrm{kg})$ or saline into mice, which were killed after 90 min. We isolated the brains, which were immunohistochemically stained for c-Fos as a biomarker for neuronal activation and assessed the expression profile of c-Fos in various sleep-arousal brain areas. Results: Our findings revealed that there were no statistically significant differences in the expression of c-Fos in the nucleus accumbens and ventrolateral preoptic area, the vertical limb of the diagonal band nucleus, horizontal limb of the diagonal band nucleus, ventral tuberomammillary nucleus, ventral tegmental area, and dorsal raphe nucleus relative to saline between saline and spinosin-treated mice. Unlike saline, spinosin markedly decreased c-Fos expression in the lateral hypothalamic area (LHA) as well as the locus coeruleus (LC). Compared to the saline injection, the application of spinosin also resulted in a marked decrease in c-Fos expression in the LHA orexin neurons.

Conclusions: These findings suggest that spinosin administration results in a restricted pattern of c-Fos expression within the LHA orexin neurons and the $L C$, suggesting that this particular neuronal inactivation contributes to sedation and hypnosis. (Folia Morphol 2020; 79, 3: 429-437)
\end{abstract}

Key words: spinosin, immunohistochemistry, lateral hypothalamic area, locus coeruleus, orexin

\section{INTRODUCTION}

Samen Ziziphi spinosae is a traditional tranquilizing medicine that has been extensively employed for the treatment of insomnia $[8,28]$. Spinosin, which is one of the major components of Samen Ziziphi spinosae, has been shown to significantly potenti- ate the hypnotic effects of pentobarbital in mice at both 10 and $15 \mathrm{mg} / \mathrm{kg}$ by decreasing sleep laten$c y$, increasing sleep time, and increasing the rate of sleep onset induced by a subhypnotic dose of pentobarbital [24]. Spinosin exerted an anxiolytic-like effect at $5 \mathrm{mg} / \mathrm{kg}$ in the elevated plus maze,

Address for correspondence: L. Chu, PhD, Department of Physiology, Basic Medical, College, Zhejiang Chinese Medical University, Hangzhou 310053, China, tel: +86-0571-86613615, e-mail: chulisheng@21cn.com 
light/dark box test, and open field test of mice [9]. In addition, the application of spinosin (15 mg/kg) to pentobarbital-treated rats significantly induces rapid eye movement (REM) sleep through the postsynaptic serotonin $1 \mathrm{~A}\left(5-\mathrm{HT}_{1 \mathrm{~A}}\right)$ receptors [25], but whether spinosin-inducing sleep is mediated via $5-\mathrm{HT}_{1 \mathrm{~A}}$ receptors remains unclear. Spinosin also induces sleep by disrupting the wake-generating systems or by increasing the activity of sleep-promoting systems.

To identify the neuronal populations and pathways by which spinosin enhances sedation and hypnosis, mice were injected with spinosin $(15 \mathrm{mg} / \mathrm{kg})$, and then neuronal activation patterns in the sleep-arousal brain areas - including the accumbens (Acb) nucleus, vertical limb of the diagonal band (VDB) nucleus, horizontal limb of the diagonal band (HDB) nucleus, ventrolateral preoptic area (VLPO), lateral hypothalamic area (LHA), ventral tuberomammillary (VTM) nucleus, ventral tegmental area (VTA), dorsal raphe (DR) nucleus, and locus coeruleus (LC) - were assessed using single c-Fos immunohistochemistry followed by dual immunostaining for c-Fos and transmitter-specific markers in the neurons of sleep-arousal brain areas based on single c-Fos immunohistochemical positive results in the sleep-arousal brain.

\section{MATERIALS AND METHODS}

\section{Animals}

Ten pathogen-free adult male mice (Shanghai SLAC Laboratory Animal Co., Ltd., China) with weights within the range of 24 to $28 \mathrm{~g}$ were maintained in a room with conditions of $20-22^{\circ} \mathrm{C}$ and light-regulated conditions ( $12 \mathrm{~h}$ light starting at 07:00 $\mathrm{h}$ ). The mice were provided ad libitum access to food and water. The experiment was performed after allowing the animals to acclimate to the laboratory conditions for at least 1 week. All efforts were made to minimise the number of animals utilised in this study and any pain and discomfort experienced by the mice. The protocol for animal handling was approved and followed the animal guidelines established by the Laboratory Animal Research Centre of Zhejiang Chinese Medical University, Hangzhou, in Zhejiang province.

\section{Spinosin injection procedures and tissue preparation}

The mice were maintained in cages throughout the entire experiment, except when they received spinosin or saline injection. Five mice were injected i.p. with $15 \mathrm{mg} / \mathrm{kg}$ of spinosin (National Institutes for Food and Drug Control, China; purity: $95.4 \%$ ) or saline. The spinosin doses were selected based on previous studies [24,25], which consistently showed that spinosin administered i.p. at a concentration of $15 \mathrm{mg} / \mathrm{kg}$ generated a significant increase in sleep time. Spinosin was mixed in a sterile saline solution at a dose of $15 \mathrm{mg} / \mathrm{mL}$, and the mice were injected with a volume of $1 \mathrm{~mL} / \mathrm{kg}$ body weight. The injections were administered at 19:00 h. After injection, the mice were immediately placed back in their cages, and their behaviour was monitored every $30 \mathrm{~min}$. Approximately $90 \mathrm{~min}$ after injection, the mice were subjected to anaesthesia using chloral hydrate $(500 \mathrm{mg} / \mathrm{kg}$, i.p.). Upon confirmation that the mice were unresponsive to tail- or foot-pinching, they were perfused with saline and then fixed with $4 \%$ paraformaldehyde. After perfusion at $4^{\circ} \mathrm{C}$ for $2 \mathrm{~h}$, their brains were isolated, post-fixed overnight in the same fixative, and then transferred to $20 \%$ sucrose. Three sets of 30- $\mu \mathrm{m}$ sections were prepared using a Leica freezing microtome (Leica, type 820-II) at the coronal plane, mounted in a cryoprotectant solution, and the stored at $-20^{\circ} \mathrm{C}$ until immunohistochemical staining.

\section{Immunohistochemical procedures}

The first set of stored free-floating tissue sections was rinsed in phosphate buffered saline (PBS), washed with $3 \% \mathrm{H}_{2} \mathrm{O}_{2}$ in PBS for 30 min, blocked with $5 \%$ goat serum (Cat. No. S-1000, Vector Laboratories, Tucson, AZ, USA) and $0.3 \%$ Triton X-100 in PBS for $1 \mathrm{~h}$ at room temperature, and subsequently incubated overnight at $4^{\circ} \mathrm{C}$ with c-Fos polyclonal rabbit antiserum (1:5,000 dilution, Cat. No. PC38, Calbiochem, Gibbstown, NJ, USA) for $48 \mathrm{~h}$ at $4^{\circ} \mathrm{C}$. The tissue sections were then incubated with a biotinylated goat anti-rabbit IgG antibody (Vector Laboratories; Cat. No. BA-1000), followed by avidin-biotin-horseradish peroxidase complex $(A B C)$ solution (Vectastain Elite ABC Kit; Vector Laboratories; Cat. No. PK-4000). The sections were rinsed with PBS and then visualised with diaminobenzidine (DAB) in the presence of nickel ammonium sulphate, which yielded a black-purple reaction product within the nuclei. Then, the sections were rinsed with PBS, placed on gelatin-coated slides, air-dried overnight, dehydrated across an ascending alcohol gradient, cleared in xylene, and coverslipped.

The other two batches of sections were processed for double immunofluorescence staining for c-Fos and transmitter-specific markers to determine major sleep-arousal-promoting neuronal groups. The sections were rinsed with PBS, blocked with 5\% donkey serum (Cat. No. 017-000-121, Jackson Immuno- 
Research Laboratories, Inc., West Grove, PA, USA) with $0.3 \%$ Triton $\mathrm{X}-100$ in PBS for $1 \mathrm{~h}$ at room temperature, and then incubated with the primary antiserum for $24 \mathrm{~h}$ at $4^{\circ} \mathrm{C}$. The primary antibodies for transmitter-specific markers consisted of a polyclonal rabbit anti-c-Fos $(1: 5,000$, Cat. No. PC38, Calbiochem) and/or a polyclonal goat anti-orexin $(1: 1,000$, Cat. No. sc-8070, Santa Cruz Biotechnology, Santa Cruz, CA, USA) to identify orexin neurons within the lateral hypothalamic area and a polyclonal rabbit anti-c-Fos (1:5000, Cat. No. PC38, Calbiochem)/ /mouse anti-tyrosine hydroxylase (TH) (1:2,000, Cat. No. ab129991, Abcam, Cambridge, UK) to identify dopaminergic neurons within the VTA and norepinephrine neurons within the LC. After incubation with the primary antiserum, the sections were rinsed with PBS and then incubated with a secondary antibody (Alexa Fluor 488-conjugated donkey anti-rabbit IgG [1:1,000, Cat. No. A21206, Invitrogen, Carlsbad, CA, USA], Alexa Fluor 594 conjugated-donkey anti-goat IgG [1:1,000, Cat. No. 705-585-147, Jackson ImmunoResearch Laboratories, Inc., West Grove, PA, USA], Alexa Fluor 594 conjugated-donkey anti-mouse IgG [1:1,000, Cat. No. A21203, Invitrogen]) for $2 \mathrm{~h}$ at room temperature. The sections were rinsed with PBS, mounted with Fluoromount-G mounting medium (SouthernBiotech, Birmingham, AL, USA), and then coverslipped. Negative controls without the primary antibody were prepared in all experiments and did not exhibit any immunoreactivity.

\section{Cell counts}

The pattern of c-Fos immunoreactivity within the major brain regions of the sleep-arousal system and other brain regions showed significant changes after treatment with spinosin. The sections were assessed, and images were captured using an Olympus IX71 microscope equipped with fluorescent and brightfield optics. We employed Adobe Photoshop CS2 in post-processing the digital photomicrographs as well as in preparing the schematic illustrations. Adjustment of sharpness, colour balance, and contrast was performed to obtain an optimal match of immunohistochemically stained cells against the background. The anatomical landmarks and abbreviations employed in this study were based on a mouse brain atlas [14]. The outlines of the sections and major structures were evaluated at low magnification $(4 \times)$, followed by mapping the profiles of the c-Fos immunoreactive (IR) neurons using high magnification.
Processing and quantification of c-Fos IR-positive neurons were conducted by a treatment-blinded examiner using ImageJ software (National Institutes of Health, Bethesda, MD, USA). Bilateral counts of all nuclei were performed on five consecutive sections. The number of c-Fos-stained nuclei and orexin- or $\mathrm{TH}$-stained soma was independently counted in tissue sections at representative rostral, middle, as well as caudal planes of the lateral hypothalamic area or VTA or LC. The density of the c-Fos + orexin (or TH) IR neurons was measured using ImageJ.

\section{Data analysis}

The results of this study are presented as the mean \pm standard error of the mean (SEM). These ratios are shown as the percentage of the orexin or $\mathrm{TH}$ cell populations that was doubled-stained for c-Fos. We used the paired $t$ test to detect differences, and $p$-values $<0.05$ were deemed statistically significant.

\section{RESULTS}

c-Fos immunoreactivity in major brain areas of the mouse sleep-arousal system after injecting spinosin or saline

The number of cells that were immunopositive for c-Fos was determined in the sleep-arousal brain regions, including Acb, VDB, HDB, VLPO, LHA, VTM, VTA, DR, as well as LC (Figs. 1, 2). There were no statistically significant differences in the number of c-Fos-IR neurons in the Acb and VLPO and decreases in the number of c-Fos-IR in the VDB, HDB, VTM, VTA, and DR between saline and spinosin-injected mice (Table 1). The LHA and LC of saline-treated mice contained a large number of c-Fos-IR, while c-Fos expression was markedly decreased in the LHA and LC following injection of spinosin into mice $(p<0.01$, Table 1$)$.

\section{Spinosin inhibits c-Fos ${ }^{+} /$orexin $^{+}$expression in the LHA}

The orexin neurons within the LHA are generally active when an animal is awake, particularly during motor activity as the animal actively explores its immediate environment. The noradrenergic cell groups of the LC are major components comprising the ascending arousal system. We next aimed to examine the expression of c-Fos ${ }^{+} /$orexin $^{+}$in the LHA and c-Fos ${ }^{+} / \mathrm{TH}^{+}$(noradrenergic neuron marker) in the LC following injection of spinosin and saline into mice. Unlike saline, spinosin inhibited the expression of c-Fos ${ }^{+} /$orexin ${ }^{+}$in the LHA (Fig. 3). c-Fos ${ }^{+} / \mathrm{TH}^{+}$expres- 

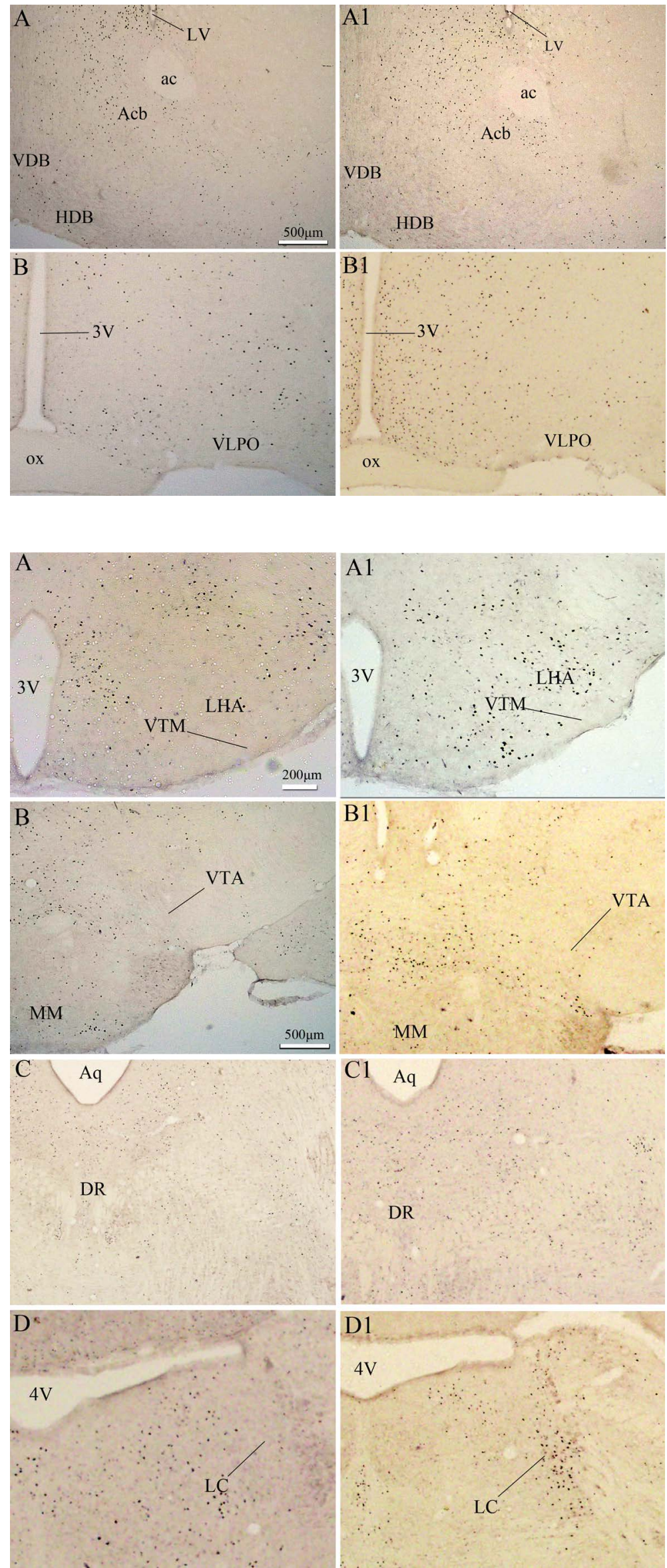

Figure 1. Effects of spinosin on key sleep/wake regulatory areas. c-Fos expression in nucleus accumbens (Acb), nuclei of the vertical limb of the diagonal band (VDB), nuclei of the horizontal limb of the diagonal band (HDB), and ventrolateral preoptic area (VLPO) following injections of spinosin (A, B) or saline (A1, B1). Scale bar $=500 \mu \mathrm{m} ; 3 \mathrm{~V}$ - third ventricle; ac - anterior commissural nucleus; LV — lateral ventricle; ox - optic chiasm.

Figure 2. Effects of spinosin on key sleep/wake regulatory areas. c-Fos expression in lateral hypothalamic area (LHA), ventral tuberomammillary nucleus (VTM), ventral tegmental area (VTA), dorsal raphe nucleus (DR), and locus coeruleus (LC) following injections of spinosin (A, B, C, D) or saline (A1, B1, C1, D1). Scale bar $=100 \mu \mathrm{m}$ in $\mathrm{A}, \mathrm{A} 1$ and $500 \mu \mathrm{m}$ in $\mathrm{B}, \mathrm{B} 1, \mathrm{C}, \mathrm{C} 1, \mathrm{D}, \mathrm{D} 1$; $3 \mathrm{~V}$ - third ventricle; $4 \mathrm{~V}$ - fourth ventricle; $\mathrm{Aq}$ - aqueduct; $f$ - fornix; $\mathrm{MM}$ - medial mammillary nucleus, medial part. 
Table 1. Counts of c-Fos-IR neurons in key brain regions of the sleep-arousal system in mice following injection of spinosin or saline*

\begin{tabular}{lcc}
\hline Brain region & Saline & Spinosin \\
\hline $\mathrm{N}$ & 5 & 5 \\
Nucleus accumbens & $51.39 \pm 10.72$ & $57.81 \pm 11.43$ \\
$\begin{array}{l}\text { Nucleus of the vertical limb of } \\
\text { the diagonal band }\end{array}$ & $23.57 \pm 4.03$ & $22.43 \pm 4.41$ \\
$\begin{array}{l}\text { Nucleus of the horizontal limb of } \\
\text { the diagonal band }\end{array}$ & $17.21 \pm 3.49$ & $16.31 \pm 3.30$ \\
$\begin{array}{l}\text { Ventrolateral preoptic area } \\
\text { Lateral hypothalamic area }\end{array}$ & $9.51 \pm 2.62$ & $9.83 \pm 2.14$ \\
$\begin{array}{l}\text { Ventral tuberomammillary } \\
\text { nucleus }\end{array}$ & $5.04 \pm 1.33$ & $3.93 \pm 1.15$ \\
Ventral tegmental area & $25.41 \pm 3.75$ & $23.17 \pm 3.49$ \\
Dorsal raphe nucleus & $10.04 \pm 2.39$ & $8.94 \pm 2.05$ \\
Locus coeruleus & $91.05 \pm 11.49$ & $40.43 \pm 5.62^{\#}$ \\
\hline
\end{tabular}

${ }^{*}$ Each value represents a mean \pm standard error of the mean of the number of labelled neurons per section per side of the brain that was averaged across animals. " $\mathrm{N}$ " indicates the number of animals. The difference ${ }^{\#} p<0.01$ of T-test is compared to saline.

sion was not observed within the LC (Fig. 4). Paired $t$ test analysis indicated that LHA c-Fos ${ }^{+} /$orexin $^{+}$expression was significantly lower after spinosin injection compared to saline injection ( $p<0.01$ ) (Fig. 5).

\section{DISCUSSION}

Injecting spinosin at a dose of $15 \mathrm{mg} / \mathrm{kg}$ did not result in an obvious change in the number of $c$-Fos-IR neurons within the Acb, VDB, HDB, VLPO, VTM, VTA, and $D R$ relative to saline injection. Spinosin injection significantly decreased c-Fos expression in the LHA and LC. In addition, spinosin also induced a significant decrease in the expression of c-Fos in the LHA orexin neurons. This distinct response patterns to spinosin suggested that spinosin induces sedation and hypnosis by activating specific neuronal populations.

\section{Methodological assessment}

c-Fos immunohistochemistry detects the activation of specific neurons activated in response to different experimental conditions; however, this technique is also associated with certain limitations. c-Fos expression is induced by an increase in cAMP or calcium levels, which commonly occur upon synaptic activation [22]. However, not all activated neurons express c-Fos, and cells that are inhibited might not express c-Fos $[3,23]$. Spinosin may influence neuronal populations that are often overlooked by immunohistochemistry. This approach enables the analysis of the correlation between neuronal activity and behavioural state, although we were unable to determine whether c-Fos expression in the LHA and LC that was inhibited by spinosin was the cause or the consequence of sleep. Further investigations on lesions in the LHA and LC in mice may further assess whether the LHA and LC neurons are essential to spinosin-induced sedation and hypnosis. Here, c-Fos in the Acb, VDB, HDB, and VLPO did not exhibit a significant increase in immunoreactivity after spinosin injection, even though these regions remain active during sleep. The mechanism underlying the lack of substantial increases in c-Fos expression remains unclear, although neuronal discharge patterns and the recruitment of certain intracellular signalling pathways could contribute to c-Fos induction. The present study employed intraperitoneal injections of spinosin to avoid cannula injury, as trauma-induced c-Fos usually occurs within $200 \mathrm{Wm}$ of the cannula track [19]. Furthermore, the number of c-Fos-IR nuclei in certain brain areas may vary extensively among consecutive sections due to size or shape variations within a cell group. Thus, for each brain region, bilateral cell counts using 10 consecutive sections were conducted to minimise sampling error. During our visualisation of double immunostaining of c-Fos and orexin/noradrenergic neurons, we were unable to distinguish whether c-Fos expression in a neuron was a direct consequence of spinosin in the neurons or an indirect result of the stimulatory effects of spinosin [2]. However, the other functions of spinosin did not coincide with the c-Fos expression patterns in the LHA and LC. Thus, in the present study, c-Fos expression in LHA orexin neurons and LC noradrenergic neurons may be the direct result of spinosin injection or the consequence of indirect activation.

\section{c-Fos immunoreactivity patterns induced by spinosin injections}

$A c b$, which is located within the rostrobasal forebrain, exhibits GABAergic projections toward a wide range of targets, such as the ventral pallidum, the LHA, the parabrachial nucleus, and the VTA, which may contribute to wakefulness $[6,13,27]$. VLPO neurons secrete inhibitory neurotransmitters, galanin, and GABA and are mainly active during sleep and send efferent projections to various major cell groups within the hypothalamus and brainstem that are involved in arousal [11, 21]. The VDB and HDB, which are located within the basal forebrain, consist of cholinergic neurons, and c-Fos immunoreactivity 

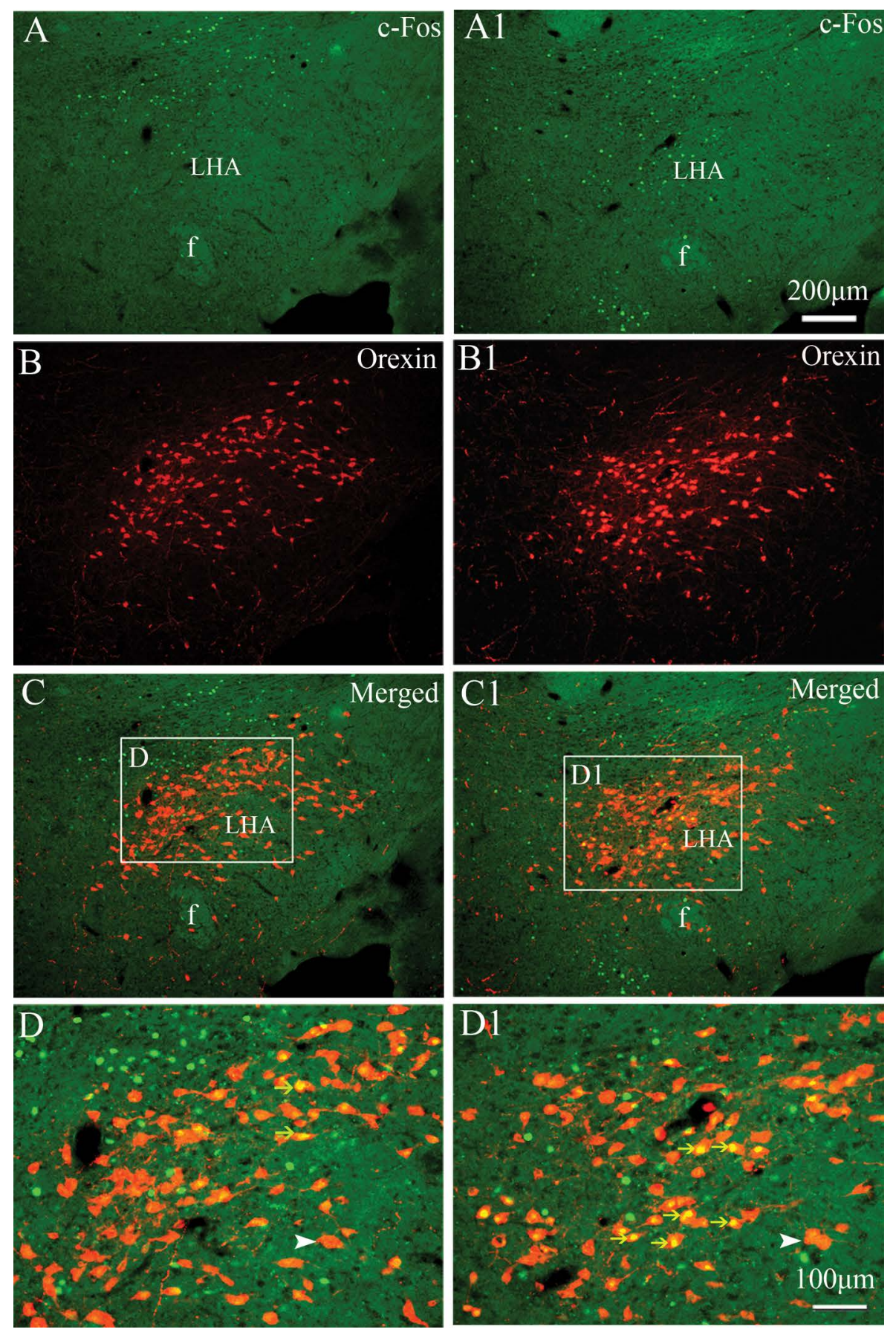

Figure 3. Photomicrographs of sections showing dual immunostaining for $\mathrm{C}-\mathrm{Fos}(\mathbf{A}, \mathbf{A} \mathbf{1}$, green) and orexin (B, B1, red) in the lateral hypothalamic area (LHA) after injecting spinosin (A, B, C, D) or saline (A1, B1, C1, D1). Yellow arrowheads show double-labelled cells, and white arrowheads show examples of single-labelled orexin cells. Most orexin-immunoreactive (IR) neurons were positively immunostained for c-Fos

(A1, B1, C1, D1) after saline injection, but most orexin-IR neurons were c-Fos-negative following spinosin injection

(A, B, C, D). Scale bar $=200 \mu \mathrm{m}$ in $\mathrm{A}, \mathrm{A} 1, \mathrm{~B}, \mathrm{~B} 1, \mathrm{C}, \mathrm{C} 1$ and $100 \mu \mathrm{m}$ in $\mathrm{D}, \mathrm{D} 1 ; \mathrm{F}$ - fornix.

has been reported during wakefulness but found to be significantly lower during sleep $[5,20]$. The VTM (histamine neuron), VTA (dopamine neuron), DR (serotonin), and LC (noradrenergic neuron) are major components of the ascending arousal system participating in cortical arousal [18]. LHA orexin neurons form ascending projections toward the cerebral cortex and descending projections directed to the monoaminergic and cholinergic cell groups of the arousal systems that are highly active during wakefulness $[4,15]$. Here, spinosin did not induce an obvious change in c-Fos-IR neuron counts in the Acb, VLPO, VDB, HDB, VTM, VTA, and DR relative to saline. In addition, spinosin significantly decreased c-Fos expression in the LHA orexin neurons and the LC. These results indicate that spinosin induces sleep by repressing the wake-generating systems or by enhancing the activity of sleep-promoting systems.

\section{How might spinosin induce behavioural sleep?}

The aim of the present study was to determine whether the inhibition of arousal-promoting neu- 

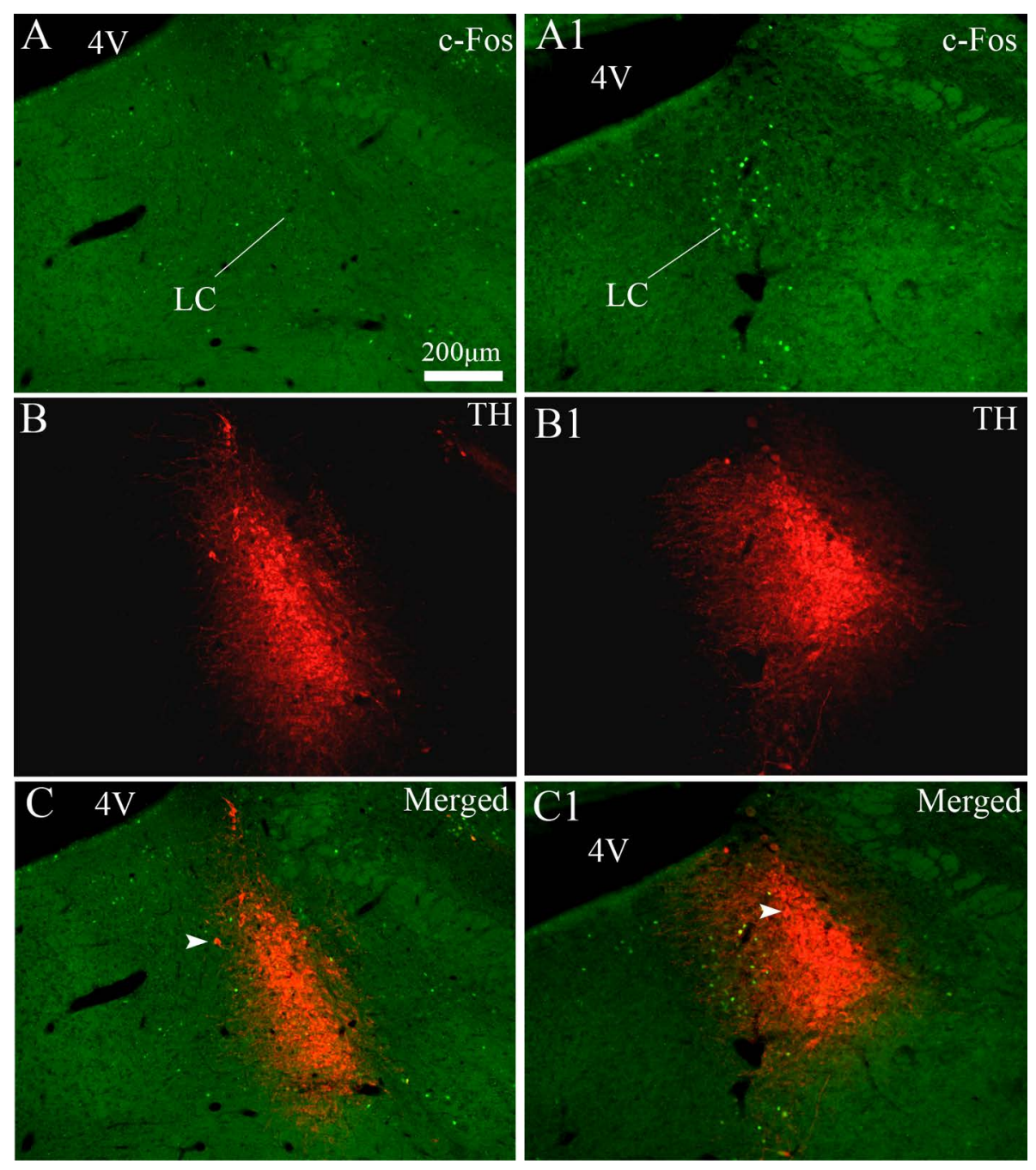

Figure 4. Photomicrographs of sections showing dual immunostaining for $\mathrm{C}$-Fos (A, A1, green) and orexin (B, B1, red) in the locus coeruleus (LC) after injecting spinosin (A, B, C) or saline (A1, B1, C1). White arrowheads indicate examples of single-labelled neurons of noradrenergic cells. No noradrenergic cells are positive for c-Fos following injections of spinosin and saline. Scale bar $=200 \mu \mathrm{m}$; $4 \mathrm{~V}$ - fourth ventricle.

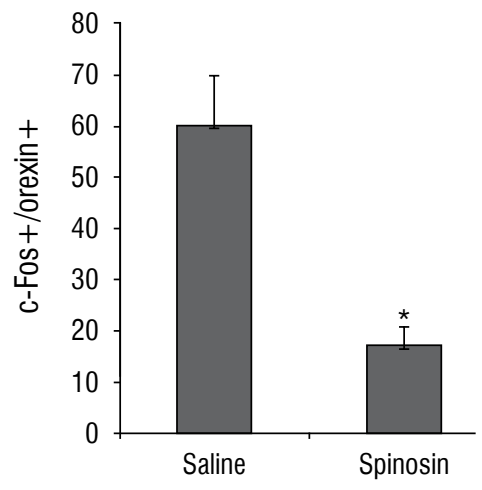

Figure 5. Number (mean \pm standard error of the mean) of dual-labelled c-Fos/orexin-IR in the lateral hypothalamic area after saline and spinosin injection. Difference from saline: ${ }^{*} p<0.01$.

rons or brain regions is correlated with behavioural sleep after spinosin injection. We determined that spinosin markedly downregulated c-Fos in the LHA and LC as well as in the LHA orexin neurons. 5- $\mathrm{HT}_{1 \mathrm{~A}}$ receptor expression was observed in serotonergic neurons as well as in LHA orexin neurons [10, 26]. The serotonergic inhibitory signals can hyperpolarise LHA orexin neurons via the $5-\mathrm{HT}_{1 \mathrm{~A}}$ receptor, which is involved in the control of sleep-wakefulness states [12]. The $5-\mathrm{HT}_{1 \mathrm{~A}}$ receptors are also distributed across the LC, with LC noradrenaline metabolism induced via their activation $[1,16]$. The $5-\mathrm{HT}_{1 \mathrm{~A}}$ receptor agonist enhanced noradrenaline metabolism in the LC, which in turn increased the firing rate of this particular brain region [17]. An earlier study revealed that spinosin (15 mg/kg, i.g.) significantly antagonizes the 5- $\mathrm{HT}_{1 \mathrm{~A}}$ agonist 8-OH-DPAT-induced decrease in total sleep time, NREM sleep, as well as REM sleep in pentobarbital-treated animals [25]. Furthermore, the combined treatment of spinosin and the $5-\mathrm{HT}_{1 \mathrm{~A}}$ antagonist resulted in significant augmentative effects on pentobarbital-induced sleep [25]. These findings suggest that spinosin could be an antagonist to the postsynaptic $5-\mathrm{HT}_{1 \mathrm{~A}}$ receptors [7]. 


\section{CONCLUSIONS}

The results of the present study indicate that spinosin can impart inhibitory effects on the firing of the LHA orexin neurons and the LC neurons. This study provides important insights into the function of spinosin in regulating sedation and hypnosis.

\section{Acknowledgements}

The Zhejiang Natural Science Fund Project (LY17H280005) and Science and Technology Innovation Team of the Basic Medical College of Zhejiang Chinese Medicine University (JCIT2016-2) supported this study.

\section{REFERENCES}

1. Clement HW, Gemsa D, Wesemann W. Serotonin-norepinephrine interactions: a voltammetric study on the effect of serotonin receptor stimulation followed in the N. raphe dorsalis and the Locus coeruleus of the rat. J Neural Transm Gen Sect. 1992; 88(1): 11-23, doi: 10.1007/BF01245033, indexed in Pubmed: 1374260.

2. Deurveilher S, Lo H, Murphy JA, et al. Differential c-Fos immunoreactivity in arousal-promoting cell groups following systemic administration of caffeine in rats. J Comp Neurol. 2006; 498(5): 667-689, doi: 10.1002/cne.21084, indexed in Pubmed: 16917819.

3. Dragunow M, Faull R. The use of c-fos as a metabolic marker in neuronal pathway tracing. J Neurosci Methods. 1989; 29(3): 261-265, doi: 10.1016/0165-0270(89)90150-7, indexed in Pubmed: 2507830.

4. Furutani N, Hondo M, Kageyama $\mathrm{H}$, et al. Neurotensin co-expressed in orexin-producing neurons in the lateral hypothalamus plays an important role in regulation of sleep/wakefulness states. PLoS One. 2013; 8(4): e62391, doi: 10.1371/journal. pone.0062391, indexed in Pubmed: 23620827.

5. Greco MA, Lu J, Wagner D, et al. c-Fos expression in the cholinergic basal forebrain after enforced wakefulness and recovery sleep. Neuroreport. 2000; 11(3): 437-440, doi: 10.1097/00001756-200002280-00002, indexed in Pubmed: 10718290.

6. Jongen-Rêlo AL, Voorn P, Groenewegen HJ. Immunohistochemical characterization of the shell and core territories of the nucleus accumbens in the rat. Eur J Neurosci. 1994; 6(8): 1255-1264, doi: 10.1111/j.1460-9568.1994. tb00315.x, indexed in Pubmed: 7526940.

7. Jung InHo, Lee HE, Park SeJ, et al. Ameliorating effect of spinosin, a C-glycoside flavonoid, on scopolamine-induced memory impairment in mice. Pharmacol Biochem Behav. 2014; 120: 88-94, doi: 10.1016/j.pbb.2014.02.015, indexed in Pubmed: 24582850.

8. Liu J, Chen Bo, Yao S. Simultaneous analysis and identification of main bioactive constituents in extract of Zizyphus jujuba var. sapinosa (Zizyphi spinosi semen) by high-performance liquid chromatography-photodiode array detection-electrospray mass spectrometry. Talanta. 2007; 71(2): 668-675, doi: 10.1016/j.talanta.2006.05.014, indexed in Pubmed: 19071358.
9. Liu J, Zhai WM, Yang YX, et al. GABA and 5-HT systems are implicated in the anxiolytic-like effect of spinosin in mice. Pharmacol Biochem Behav. 2015; 128: 41-49, doi: 10.1016/j. pbb.2014.11.003, indexed in Pubmed: 25449359.

10. Mineur YS, Einstein EB, Bentham MP, et al. Expression of the 5-HT1A serotonin receptor in the hippocampus is required for social stress resilience and the antidepressant-like effects induced by the nicotinic partial agonist cytisine. Neuropsychopharmacology. 2015; 40(4): 938-946, doi: 10.1038/npp.2014.269, indexed in Pubmed: 25288485.

11. Moore JT, Chen J, Han Bo, et al. Direct activation of sleep-promoting VLPO neurons by volatile anesthetics contributes to anesthetic hypnosis. Curr Biol. 2012; 22(21): 2008-2016, doi: 10.1016/j.cub.2012.08.042, indexed in Pubmed: 23103189.

12. Muraki Yo, Yamanaka A, Tsujino N, et al. Serotonergic regulation of the orexin/hypocretin neurons through the 5-HT1A receptor. J Neurosci. 2004; 24(32): 7159-7166, doi: 10.1523/JNEUROSCI.1027-04.2004, indexed in Pubmed: 15306649.

13. Oishi Yo, Xu Qi, Wang Lu, et al. Slow-wave sleep is controlled by a subset of nucleus accumbens core neurons in mice. Nat Commun. 2017; 8(1): 734, doi: 10.1038/ s41467-017-00781-4, indexed in Pubmed: 28963505.

14. Paxinos G. Franklin, KBJ. The Mouse Brainin Stereotaxic Coordinates. San Diego: Academic Press. 1997.

15. Peyron C, Tighe DK, van den Pol AN, et al. Neurons containing hypocretin (orexin) project to multiple neuronal systems. J Neurosci. 1998; 18(23): 9996-10015, indexed in Pubmed: 9822755.

16. Rioja J, Santín $\sqcup$, López-Barroso D, et al. 5-HT1A receptor activation counteracted the effect of acute immobilization of noradrenergic neurons in the rat locus coeruleus. Neurosci Lett. 2007; 412(1): 84-88, doi: 10.1016/j.neulet.2006.10.013, indexed in Pubmed: 17197087.

17. Sanghera MK, McMillen BA, German DC. Buspirone, a non-benzodiazepine anxiolytic, increases locus coeruleus noradrenergic neuronal activity. Eur J Pharmacol. 1982; 86(1): 107-110, doi: 10.1016/0014-2999(82)90406-x, indexed in Pubmed: 6130954.

18. Saper CB, Scammell TE, Lu J. Hypothalamic regulation of sleep and circadian rhythms. Nature. 2005; 437(7063): 1257-1263, doi: 10.1038/nature04284, indexed in Pubmed: 16251950 .

19. Scammell TE, Gerashchenko DY, Mochizuki T, et al. An adenosine A2a agonist increases sleep and induces Fos in ventrolateral preoptic neurons. Neuroscience. 2001; 107(4): 653-663, doi: 10.1016/s0306-4522(01)00383-9, indexed in Pubmed: 11720788.

20. Schrader JA, Smale L, Nunez AA. Pregnancy affects FOS rhythms in brain regions regulating sleep/wake state and body temperature in rats. Brain Res. 2012; 1480: 53-60, doi: 10.1016/j.brainres.2012.09.003, indexed in Pubmed: 22975436 .

21. Sherin JE, Shiromani PJ, McCarley RW, et al. Activation of ventrolateral preoptic neurons during sleep. Science. 1996; 271(5246): 216-219, doi: 10.1126/science.271.5246.216, indexed in Pubmed: 8539624.

22. Sim L, Selley DE, Tsai KP, et al. Calcium and cAMP mediated stimulation of Fos in cultured hypothalamic tyrosine hydroxylase-immunoreactive neurons. Brain Res. 1994; 
653(1-2): 155-160, doi: 10.1016/0006-8993(94)90384-0, indexed in Pubmed: 7982047.

23. Ueta $Y$, Hara $Y$, Kitamura $K$, et al. Action sites of adrenomedullin in the rat brain: functional mapping by Fos expression. Peptides. 2001; 22(11): 1817-1824, doi: 10.1016/ s0196-9781(01)00528-9, indexed in Pubmed: 11754968.

24. Wang LE, Bai YJ, Shi XR, et al. Spinosin, a C-glycoside flavonoid from semen Zizhiphi Spinozae, potentiated pentobarbital-induced sleep via the serotonergic system. Pharmacol Biochem Behav. 2008; 90(3): 399-403, doi: 10.1016/j. pbb.2008.03.022, indexed in Pubmed: 18466960.

25. Wang LE, Cui XY, Cui SY, et al. Potentiating effect of spinosin, a C-glycoside flavonoid of Semen Ziziphi spinosae, on pentobarbital-induced sleep may be related to postsynaptic 5-HT(1A) receptors. Phytomedicine. 2010; 17(6): 404-409, doi: 10.1016/j.phymed.2010.01.014, indexed in Pubmed: 20171860
26. Zanderigo F, Pantazatos $S$, Rubin-Falcone $H$, et al. In vivo relationship between serotonin $1 \mathrm{~A}$ receptor binding and gray matter volume in the healthy brain and in major depressive disorder. Brain Struct Funct. 2018; 223(6): 2609-2625, doi: 10.1007/s00429-018-1649-6, indexed in Pubmed: 29550938.

27. Zhang JP, Xu Qi, Yuan XS, et al. Projections of nucleus accumbens adenosine $A 2 A$ receptor neurons in the mouse brain and their implications in mediating sleep-wake regulation. Front Neuroanat. 2013; 7: 43, doi: 10.3389/ fnana.2013.00043, indexed in Pubmed: 24409122.

28. Zhang $M$, Zhang $Y$, Xie J. Simultaneous determination of jujuboside $A, B$ and betulinic acid in semen Ziziphi spinosae by high performance liquid chromatography-evaporative light scattering detection. J Pharm Biomed Anal. 2008; 48(5): 1467-1470, doi: 10.1016/j.jpba.2008.09.022, indexed in Pubmed: 18977107. 